\title{
Stimulated X-ray Emission for Materials Science.
}

M. Beye ${ }^{1}$, S. Schreck ${ }^{1,3}$, F. Sorgenfrei ${ }^{1,2}$, C. Trabant ${ }^{1,3,4}$, N. Pontius ${ }^{1}$, C. Schüßler-Langeheine ${ }^{1}$, W. Wurth ${ }^{2} \&$ A. Föhlisch ${ }^{1,3}$

${ }^{1}$ Institute for Methods and Instrumentation of Synchrotron Radiation Research G-ISRR, Helmholtz-Zentrum Berlin für Materialien und Energie GmbH, Albert-Einstein-Straße 15, 12489 Berlin, Germany

${ }^{2}$ Institut für Experimentalphysik, Universität Hamburg and Centre for Free-Electron Laser Science, Luruper Chaussee 149, 22761 Hamburg, Germany

${ }^{3}$ Fakultät für Physik und Astronomie, Universität Potsdam, Karl-Liebknecht-Straße 24-25, 14476 Potsdam, Germany

${ }^{4}$ II. Physikalisches Institut, Universität zu Köln, Zülpicher Straße 77, 50937 Köln, Germany

Resonant Inelastic X-ray Scattering (RIXS) or X-ray Emission Spectroscopy (XES) access the energy and dispersion of elemental vibronic, charge, spin and orbital excitations for samples down to micron size ${ }^{1-7}$. Those low energy excitations govern functionality in matter, where small external triggers lead to drastic changes of materials properties. The achilles heel of RIXS has been the needed high photon densities on the sample to compensate for sub-percent fluorescence yields for soft $\mathrm{X}$-rays ${ }^{8}$. Thus sample damage from the dominant non-radiative decays poses limits to materials and the spectral resolution. Means to improve the yield are thus highly desirable. In this work, we report on stimulated X-ray emission for crystalline silicon at photon densities easily achievable with free-electron lasers (FELs) ${ }^{9}$. The 
stimulated radiative decay of core excited species at the expense of non-radiative processes reduces sample damage and allows for the narrow bandwidth detection in the directed beam of stimulated radiation. We deduce how stimulated X-ray emission can be enhanced by several orders of magnitude to provide with high yield and reduced sample damage a superior probe for low energy excitations and their dispersion in matter. This is the first step to bring non-linear X-ray physics in the condensed phase from theory ${ }^{10-16}$ to application.

In the soft X-ray region, the use of non-linear techniques to enhance the signal levels has so far been rendered impossible due to small cross-sections and the short life-time of core-excited states in the regime of some femtoseconds. Only in the last few years, FELs have become available, producing ultrashort, intense soft X-ray pulses ${ }^{9,17-20}$. Recently, the stimulation of emission from a single fluorescence line in a rare gas ${ }^{21}$ and hard X-ray / optical sum frequency generation ${ }^{22}$ have been demonstrated.

We present here stimulated X-ray emission from a solid state sample recorded at the freeelectron laser in Hamburg (FLASH) for non-resonant silicon $L$-edge excitation at $115 \mathrm{eV}$ photon energy. With FEL radiation we produce regions with high $2 p$ core excitation densities. The spontaneously emitted radiation from recombination of the $2 p$ core holes ( 85 to nearly $100 \mathrm{eV}$ photon energy) seeds the stimulated emission of soft X-ray photons. The emitted spectrum is determined by the spontaneous emission as observed in a typical RIXS or XES experiment and thus conserves all the information and specificity of these methods. By carefully choosing the geometry, we can significantly enhance the weak fluorescence signal at the expense of Auger decays. Fewer elec- 
trons are emitted and electronic damage to the sample is minimized. By properly shaping the FEL beam footprint on the sample the detected signal can be enhanced by orders of magnitude since the usually isotropic emission can get directed towards the detector. This opens a perspective for nonlinear spectroscopy in the X-ray region adopting concepts from non-linear optics, merged with the high information depth of X-ray spectroscopy and intrinsically able to resolve femtosecond dynamics ${ }^{10-16,23-25}$.

The absorption and stimulated emission probabilities $P$ can be commonly approximated for infinitesimally thin samples where the photons interact on a short length $d x$ as

$$
P=\sigma \rho d x
$$

with the number density $\rho$ of absorbing atoms (further subscripted ${ }_{\text {atom }}$ ) or respectively of excited atoms for stimulation (subscripted ${ }_{c h}$ for core hole). The X-ray absorption or stimulation cross-sections $\sigma$ are determined by the dipole transition matrix elements between the core level and unoccupied (absorption) or occupied (stimulated emission) valence states and are approximated at this point to be the same. The cross-section is connected with the absorption length $\lambda=\left(\sigma \rho_{\text {atom }}\right)^{-1}$. The effect of stimulated emission is large, when the stimulation probability approaches unity $P \stackrel{!}{=} 1=\sigma \rho_{c h} d x$, yielding

$$
\frac{\rho_{c h}}{\rho_{\text {atom }}}=\frac{\lambda}{d x}
$$

. For effective stimulation, the absorption length relative to the interaction lengths of stimulating photons has to be similar to the fraction of core excited atoms. For comparable interaction and absorption lengths, every atom needs to be core excited; a population inversion is needed. 
In forward direction, stimulated X-ray emission has been demonstrated in the gas phase ${ }^{21}$. In this geometry, the maximal interaction length where stimulating photons can interact with core holes, is intrinsically given by the absorption length. The sample has to absorb one X-ray photon per atom. If applied to solids, this energy usually destroys the bonding network and is hence not suited for spectroscopic studies of the undisturbed system.

The situation is very different for other geometries. Along the lateral dimension of the X-ray focus, the interaction length can be significantly larger. With soft X-rays on solids, the absorption length of the exciting radiation with an energy slightly above an absorption edge is typically tens or hundreds of nm, while the lateral dimensions of typical X-ray foci are $\mu \mathrm{m}$ to hundreds of $\mu \mathrm{m}$. The interaction length is then limited by the absorption length of the RIXS and XES signals with a photon energy below the absorption edge, which are typically an order of magnitude larger than for the exciting radiation, consequently lower excitation densities are needed, when the observation direction is chosen to be along the lateral dimension of the projected focus instead. This situation is sketched in Fig. 1, together with measured data of the energy integrated number of photons at several angles to the surface. We observe an emission maximum at around $9^{\circ}$, which is the result of a balance of the absorption length of the exciting radiation and the interaction length of the emitted radiation inside the excited volume.

For a deeper experimental analysis, we studied the emission with a spectrometer. Due to experimental constraints, it was placed at around $15^{\circ}$ to the sample surface. We observed a characteristic dependence of the total number and the spectral distribution of the outgoing photons on 
the incoming photon flux (Fig. 2 and 3).

Before we analyze the experimental data, we discuss the relevant processes that have to be considered to derive the number of stimulated photons. The explicit formulation is given in the supplementary material. Stimulated emission can be treated along the same lines as absorption. We hence can formulate a differential equation as a function of the interaction length in an Ansatz similar to the derivation of the Lambert-Beer law for absorption. This approach is inherently timeindependent. Auger decays are not explicitly treated, since Auger processes and radiative emission are independent processes and are only related through the number of core holes. Auger decays are implicitly included, though, through the core hole life time.

Since Auger decays are the only other significant decay channel of the core holes and since stimulated emission reduces the number of core holes, we expect a substantial decrease in the number of Auger processes. The effective life time of the core holes, taking all decay channels into account, gets shortened.

We formulate a differential equation for the gain in the number of observed stimulated photons. $N_{\text {stim,obs }}$ is the number of stimulated photons that get emitted into a detector with a finite acceptance angle and detection efficiency:

$$
\frac{d N_{\text {stim,obs }}}{d x}=N_{\text {obs }} \rho_{c h} \sigma_{\text {stim }}
$$

with the cross section for transitions between the respective valence and the core state $\sigma_{\text {stim }}$ (now generally different from $\left.\sigma_{a b s}\right)$. The total number of photons in the observation direction $N_{o b s}$ drive 
the observed stimulation and is the sum of the number of spontaneously emitted photons in this direction $N_{s p, o b s}$ as seed, as well as $N_{s t i m, o b s}$ itself. Without stimulation, $N_{s p, o b s}$ is proportional to the number of the incoming photons. The fact that stimulated photons are available to stimulate further leads to a non-linear increase of the stimulated signal. At low photon numbers stimulation is negligible and the spontaneous emission forms a linear lower limit for the dependence of the observed number of photons as a function of incoming photon numbers.

The next important factor is the instantaneous density of core holes $\rho_{c h}$ that are available for stimulated decays. Although every photon in an X-ray pulse gets absorbed in the sample, the instantaneous number of core holes is smaller than one per incoming photon depending on the pulse length $\tau_{p l}$ and the core hole lifetime $\tau_{l t}$ : some core holes already decay before the pulse is over. Beside through spontaneous decay, the number of core holes is reduced by each stimulated photon. To treat this effect, we split the decay rate of the core holes in two parts: the Auger decays enter through the fixed core hole life time, while the stimulation directly reduces the number of core holes. Thus the change in the number of stimulated photons depends on $N_{\text {stim,obs }}$ itself, this time with a negative sign leading to saturation.

An upper limit for stimulated emission is reached, when every core hole gets stimulated. For high incoming photon numbers, the observed number of photons will thus saturate towards an upper linear limit as a function of incoming photon numbers.

The solution of the differential equation provides the dependence of the observed number of stimulated photons on the experimental parameters. We approximate the lateral distribution of the 
incoming beam and the exponentially decaying absorption profile into the depth of the sample with a constant core hole density (albeit modified through stimulated emission), inside a cylindricallyshaped excitation volume, the size of which is given by the focal sizes and the absorption length of the incoming radiation. The interaction length for stimulation in this volume extends from the bottom of the excited cylinder to the sample surface in the direction of observation and is thus given only through the geometry of the experiment.

For soft X-rays at current FELs, focal sizes on the order of $10 \mu \mathrm{m}$ and pulse lengths around the core hole life time are well within reach. With typical absorption lengths in solids for the incoming photons of around $100 \mathrm{~nm}$ and for the emission of around $1 \mu \mathrm{m}$ and stimulation crosssections of 1 Mbarn $^{26}$, stimulation becomes important at about $10^{11}$ incoming photons per pulse. Typical soft X-ray FELs produce up to $10^{13}$ photons per shot, so that stimulated emission has to be considered as a dominating effect.

We now turn to the experimental observations. In Fig. 1.b, we show the angular dependence of the emission signal. The incidence angle is chosen to be $45^{\circ}$ and we find enhanced intensity for the specular geometry with the detector at $45^{\circ}$. Additionally, we find a strong enhancement of the signal by about a factor of five at grazing angles around $9^{\circ}$, far from the specular reflection. It appears in a narrow angular window and is indeed the result of a non-linear effect. With the used excitation conditions, we are deep in the regime where the stimulated emission saturates and the observed effects are variations in the angular distribution of the stimulated emission. The maximum signal is expected in a direction for which the interaction length inside the core excited volume 
is as large as possible with the reabsorption probability minimized. Since the absorption length for the incoming radiation is about an order of magnitude shorter than the reabsorption length, the optimal angle is expected around $\arcsin (0.1) \approx 6^{\circ}$ which is in reasonably good agreement with the experiment.

In Fig. 2.a, we show the total number of observed photons in our spectrometer placed at $15^{\circ}$ grazing to the surface, along with a fit to the expected dependence. We also include the linear limits derived from the fit. One observes that already in the lower studied fluence range, the deviation from the linearity is observed and the stimulated emission quickly saturates to the upper limit. In the chosen experimental geometry, the enhancement of the emission between purely spontaneous and saturated stimulation is only about a factor of two. This is because the majority of the stimulated photons are radiated more grazing to the surface and do not reach our detector as shown in Fig. 1.b. Still, the non-linear enhancement and saturation of the signal through stimulated emission are clearly observed.

It is instructive to display the total number of observed photons divided by the number of incoming photons (see Fig. 2.b). This yields the conversion efficiency from incoming photons to emitted photons, as seen by the detector - the detected conversion. The limiting linear dependences turn into limiting constant conversion efficiencies and are determined by the experimental parameters. The non-linearity of the curve becomes obvious as a monotonous change from the lower to the upper limit.

The photon number needed for the non-linear part between the limiting lines is directly re- 
lated also to the emission energy dependent stimulation cross-section. For larger stimulation crosssections, the non-linearity and thus the saturation of stimulated emission will occur at smaller incoming photon numbers. In Fig. 3.a, we show the spectral evolution of the silicon $L$-edge emission depending on the number of incoming photons and how we separate the spectrum into parts of different emission intensity. Since the stimulation cross-section contains the same matrix elements as the spontaneous emission probability, which is in turn proportional to the measured spectral emission intensity, the stimulation of emission becomes more effective for emission energy regions that show a high intensity already at low fluences. Fig. 3.b displays the dependence of the normalized detected conversion on the number of incoming photons in specific emission energy regions. While the peaked region around $90 \mathrm{eV}$ emission energy, as the most intense feature, approaches saturated stimulation fastest, the other regions follow in the order of spectral intensity.

The emission energy region for usually unoccupied states above the band gap shows a strikingly different behavior. Here, a secondary effect sets in. Photo emitted electrons and electrons from Auger decays scatter inelastically in the sample, very quickly creating a multitude of delocalized electron-hole pair excitations around the band gap. This effect becomes stronger with an increasing number of incoming photons and the connected radiative decays annihilate core holes that contributed to the stimulated emission of other parts of the spectrum at lower photon numbers. Therefore, the detected conversion in other parts of the spectrum actually shrinks in contrast to becoming constant. Nevertheless, since all emission processes saturate when all core holes are stimulated down, the total detected conversion still becomes constant (cf. the integral detected conversion shown in Fig. 2.b). 
In the direction of $15^{\circ}$ from the surface, the detected conversion increases by a bit more than a factor of two between the limiting cases of spontaneous emission and saturated stimulated emission. By going to a more shallow angle, we can increase the gain further by another factor of five (see the difference in the saturated emission signal in Fig. 1.b). This value is limited by the round shape of the FEL beam footprint on the sample. The shape of the beam leads to the same maximized interaction length in every azimuthal direction in the sample surface plane and thus to a stimulated emission profile which is rotational symmetric around the sample normal at the center of the beam.

The observed gain can obviously be further increased, when the footprint of the irradiated photons is specifically shaped. An elongation of the footprint in the observation direction will further enhance the observed photon numbers while the signal gets reduced in other directions. In order to ensure temporal overlap of the core excitations in the sample and the majority of the stimulated photons traveling with the speed of light, the footprint of the exciting beam needs to be elongated by placing the sample grazing to the incident beam. In this way, the exciting wave front will travel along the sample surface in time with the stimulated photons and the enhancement of the emission signal will be maximized while X-ray induced sample damage is further minimized.

The same applies to soft X-ray experiments from other systems, because the determining parameters are very similar for most materials. The signal gain that we expect as compared to normal RIXS or XES experiments stems from two sources. On one hand, the saturation of stimulated emission is an indicator that most core holes decay via the emission of photons instead of 
decaying via Auger processes. This pushes the observed fluorescence yield from the $10^{-3}$ level into the direction of unity. On the other hand, directing the majority of the emission towards the detector can substantially increase the signal, since typical fluorescence detectors (and our spectrometer as well) have a geometrical acceptance of less than $10^{-4}$ of the full solid angle. Therefore, stimulated emission can enhance the detected signal by several orders of magnitude. We note that in our experiment, we did observe traces of sample damage. Generally though, with optimized geometries at optimized X-ray sources, the signal gain can be used to minimize acquisition times in the non-damaging regime, or alternatively to record full spectra in a single X-ray shot, such that the spectroscopic information is essentially generated before X-ray induced changes set in.

The such enhanced signal levels enable, e.g. highly selective RIXS and XES studies with considerably improved energy resolution, so that new low-energy excitations could be discovered. Through the combination with pump-probe techniques huge parameter spaces in the ultrafast time domain can be quickly mapped. Most prominently, we expect a novel field of science opening through combining non-linear techniques from optical lasers with the high specificity of X-rays, so that small signals from extremely dilute active centers can be specifically dissected.

\section{Methods Summary}

Photon Parameters. The experiments have been conducted at the free-electron laser FLASH at Hamburg, Germany, operating at a photon energy of $115 \mathrm{eV}$. The spectral resolved data was recorded at a repetition rate of $5 \mathrm{~Hz}$ with $30 \mathrm{fs}_{\text {pulses }}{ }^{17,27}$. We used the grating of beam line PG 2 as a mirror ${ }^{28}$. The focus was round with $45 \mu \mathrm{m}$ diameter, leading to fluences up to $1 \mathrm{~J} / \mathrm{cm}^{2}$. The 
angular dependence was measured at beam line BL $2^{29}$ at a burst repetition rate of $10 \mathrm{~Hz}$, each including 100 pulses at $250 \mathrm{kHz}$. The focal size was adjusted to be about $40 \mu \mathrm{m}$ to compensate for the now $50 \mathrm{fs}$ long pulses. With a gas attenuator, we set the fluence around $1 \mathrm{~J} / \mathrm{cm}^{2}$. The incoming photon numbers have been measured for each shot by a gas monitor detector (GMD) ${ }^{29}$.

Experimental Setup. We scanned hydrogen passivated silicon (100) surfaces through the beam. Spectra were recorded with a Scienta XES 355 spectrometer ${ }^{30}$ and a single-photon counting, multi-hit capable detector centered at $92 \mathrm{eV}$ with a resolution of $0.4 \mathrm{eV}$. The scattering plane was the horizontal plane of polarization of the incoming photons with the spectrometer at around $85^{\circ}$ to the beam $\left(15^{\circ}\right.$ to the sample surface), while the pulses impinged under an angle of $80^{\circ}$ to the surface.

For the angular dependence, we used an avalanche photodiode with an aluminum filter to block optical light. The incidence angle was $45^{\circ}$ while the detection angle was varied.

Analysis. With our spectrometer, we recorded around 40.000 single shots. The data was sorted, binned and the standard deviation of the averaged values were computed for each bin. With the photodiode, we analyzed 15.000 bursts.

During FEL irradiation, the emission from a plasma plume was optically visible at the sample and slight ablation was observed.

1. Kotani, A. \& Shin, S. Resonant inelastic x-ray scattering spectra for electrons in solids. Rev. Mod. Phys. 73, 203-246 (2001). 
2. Schlappa, J. et al. Collective magnetic excitations in the spin ladder $S r_{14} C u_{24} O_{41}$ measured using high-resolution resonant inelastic x-ray scattering. Phys. Rev. Lett. 103, 047401 (2009).

3. Hennies, F. et al. Resonant inelastic scattering spectra of free molecules with vibrational resolution. Phys. Rev. Lett. 104, 193002 (2010).

4. Ament, L. J. P., van Veenendaal, M., Devereaux, T., Hill, J. P. \& van den Brink, J. Resonant inelastic x-ray scattering studies of elementary excitations. Rev. Mod. Phys. 83, 705 (2011).

5. Le Tacon, M. et al. Intense paramagnon excitations in a large family of high-temperature superconductors. Nat. Phys. 7, 725-730 (2011).

6. Pietzsch, A. et al. Spatial quantum beats in vibrational resonant inelastic soft x-ray scattering at dissociating states in oxygen. Phys. Rev. Lett. 106, 153004 (2011).

7. Schlappa, J. et al. Spin-orbital separation in the quasi-one-dimensional mott insulator $\mathrm{Sr}_{2} \mathrm{CuO}_{3}$. Nature 485, 82-86 (2012).

8. Krause, M. O. Atomic radiative and radiationless yields for K and L shells. J. Phys. Chem. Ref. Data 8, 307 (1979).

9. McNeil, B. W. J. \& Thompson, N. R. X-ray free-electron lasers. Nat. Photonics 4, 814-821 (2010)

10. Tanaka, S. \& Mukamel, S. Coherent x-ray raman spectroscopy: A nonlinear local probe for electronic excitations. Phys. Rev. Lett. 89, 043001 (2002). 
11. Mukamel, S. Multiple core-hole coherence in X-ray four-wave-mixing spectroscopies. Phys. Rev. B 72, 235110 (2005).

12. Schweigert, I. \& Mukamel, S. Probing valence electronic wave-packet dynamics by all x-ray stimulated raman spectroscopy: A simulation study. Phys. Rev. A 76, 012504 (2007).

13. Harbola, U. \& Mukamel, S. Coherent stimulated x-ray raman spectroscopy: Attosecond extension of resonant inelastic x-ray raman scattering. Phys. Rev. B 79, 085108 (2009).

14. Patterson, B. D. Resource letter on stimulated inelastic x-ray scattering at an XFEL. SLAC Technical Note 1-19 (2010).

15. Sun, Y.-P., Liu, J.-C., Wang, C.-K. \& Gel'mukhanov, F. Propagation of a strong X-ray pulse: Pulse compression, stimulated raman scattering, amplified spontaneous emission, lasing without inversion, and four-wave mixing. Phys. Rev. A 81, 013812 (2010).

16. Biggs, J. D., Zhang, Y., Healion, D. \& Mukamel, S. Two-dimensional stimulated resonance raman spectroscopy of molecules with broadband x-ray pulses. J. Chem. Phys. 136, 174117 (2012)

17. Ackermann, W. et al. Operation of a free-electron laser from the extreme ultraviolet to the water window. Nat. Photonics 1, 336-342 (2007).

18. Emma, P. et al. First lasing and operation of an angstrom-wavelength free-electron laser. Nat. Photonics 4, 641-647 (2010). 
19. Di Mitri, S. et al. FERMI@Elettra, a seeded free electron laser source for a broad scientific user program. In Tschentscher, T. \& Cocco, D. (eds.) Advacnces in X-ray free-electron lasers: radiation schemes, X-ray optics, and instrumentation, vol. 8078 of Proceedings of SPIE (2011).

20. Pile, D. X-rays: First light from SACLA. Nat. Photonics 5, 456-457 (2011).

21. Rohringer, N. et al. Atomic inner-shell x-ray laser at 1.46 nanometres pumped by an x-ray free-electron laser. Nature 481, 488-491 (2012).

22. Glover, T. E. et al. X-ray and optical wave mixing. Nature 488, 603-608 (2012).

23. Beye, M., Sorgenfrei, F., Schlotter, W. F., Wurth, W. \& Föhlisch, A. The liquid-liquid phase transition in silicon revealed by snapshots of valence electrons. Proc. Natl. Acad. Sci. USA 107, 16772-16776 (2010).

24. Wernet, P. Electronic structure in real time: mapping valence electron rearrangements during chemical reactions. Phys. Chem. Chem. Phys. 13, 16941-16954 (2011).

25. Salen, P. et al. Experimental verification of the chemical sensitivity of two-site double corehole states formed by an x-ray free-electron laser. Phys. Rev. Lett. 108, 153003 (2012).

26. Yeh, J. J. \& Lindau, I. Atomic subshell photoionization cross-sections and asymmetry parameters - 1 less-than-or-equal-to z less-than-or-equal-to 103. Atom. Data Nucl. Data 32, 1-155 (1985). 
27. Frühling, U. et al. Single-shot terahertz-field-driven x-ray streak camera. Nat. Photonics 3, $523-528$ (2009).

28. Martins, M. et al. Monochromator beamline for FLASH. Rev. Sci. Instrum. 77, 115108 (2006).

29. Tiedtke, K. et al. The soft X-ray free-electron laser FLASH at DESY: beamlines, diagnostics and end-stations. New J. Phys. 11, 023029 (2009).

30. Nordgren, J. Special issue - Soft x-ray emission spectroscopy - Preface. J. Electron Spectrosc. 110, IX-X (2000).

Acknowledgements We want to thank Nina Rohringer, Andreas Scherz and Jo Stöhr for stimulating discussions. We acknowledge support from the FLASH staff. Financial support was given to M.B. by the VolkswagenStiftung. Further support was given by the Germany federal ministry of education and research through the priority program FLASH: Matter in the light of ultrashort and extremely intense x-ray pulses and through contract 05K10PK2 and by the Deutsche Forschungsgemeinschaft through the graduate school: Physics with new advanced coherent radiation sources.

Author Contributions All authors contributed to planning parts of the experiment. Research was conducted by F.S., C.T., N.P., C.S.-L. and M.B. Data analysis and interpretation was done by M.B., S.S. and A.F. The formalism was developed and the manuscript was written by M.B. with input from all co-authors.

Reprints Reprints and permissions information is available at www.nature.com/reprints.

Competing Interests The authors declare that they have no competing financial interests. 
Correspondence Correspondence and requests for materials should be addressed to M.B. (email: martin.beye@helmholtz-berlin.de). 


\section{N}

N.

Non

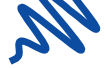

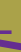

2

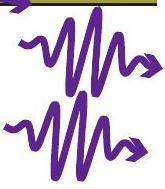

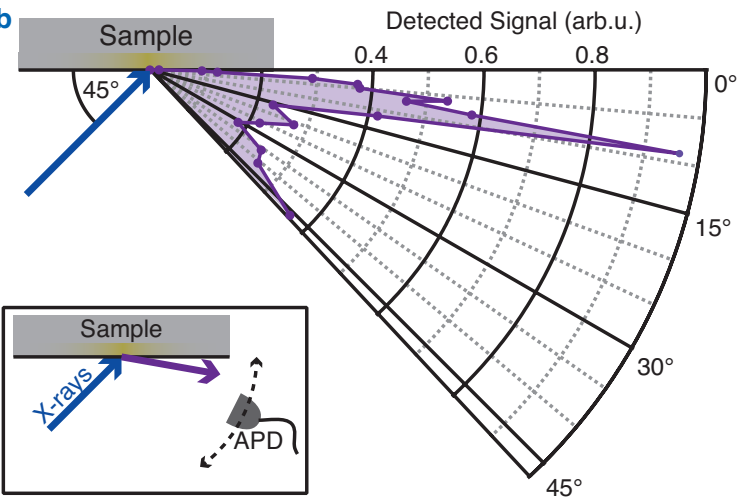



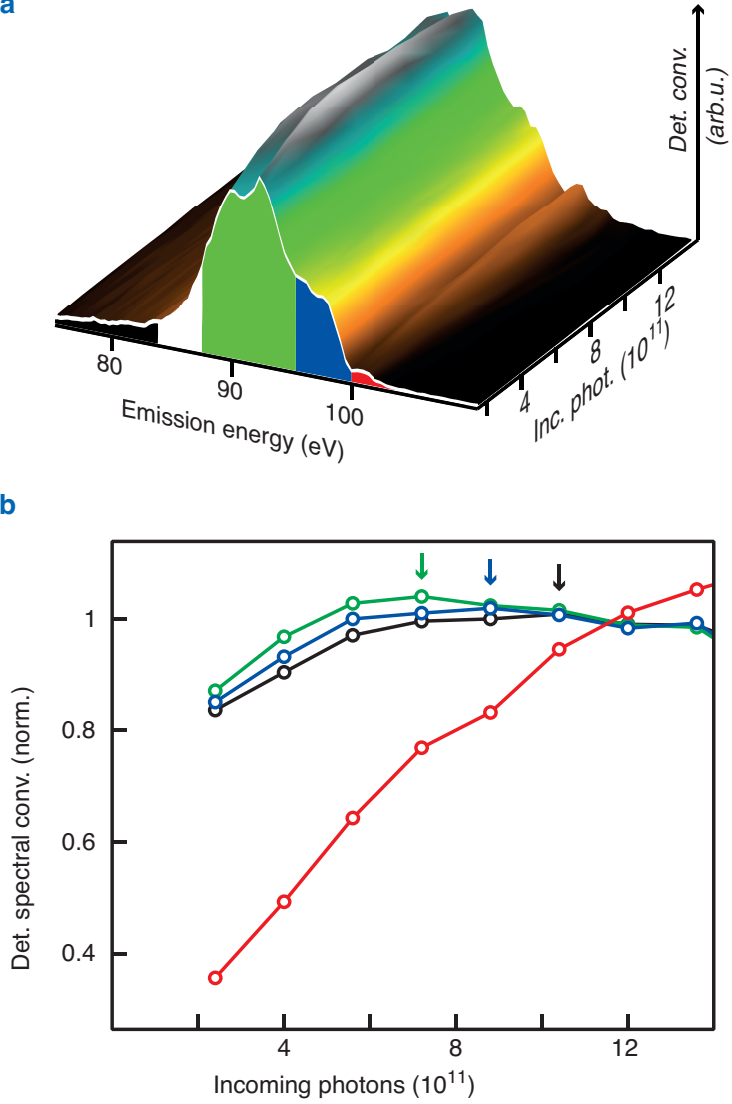
Figure 1 Geometry to observe spontaneously stimulated X-ray emission from solids.

(a) X-rays create core-excitations in the solid (yellow). A cascade of stimulated emission builds up in a direction where the penetration depth $\lambda$ of incoming photons is balanced with the absorption length $L$ for emission.

(b) The total emission is detected as a function of glancing angle at fluences where stimulated emission saturates. An enhancement is observed for shallow angles, where the interaction length for emitted photons is longest. This direction is far away from the specular reflection increase around $45^{\circ}$. The inset displays the arrangement of the experiment.

Figure 2 Observing stimulated emission from a solid.

(a) Red symbols show the measured number of counts per shot versus the number of incoming photons. The error bars are one standard deviation of the averaged values. The grey area is between the limiting cases of spontaneous emission and saturation of stimulated emission. The green line is a fit to the derived formula. The inset shows the geometry of the experiment.

(b) The same data is plotted as detected conversion, after dividing the detector counts by the number of incoming photons. The linear limits now become constants.

Figure 3 Spectrally resolved stimulated emission.

(a) The detected conversion is shown, highlighting the integration regions for data plotted in (b). 
(b) The maximum detected conversion for different spectral regions is marked with an arrow. The most intense features saturate first (green, $87-95 \mathrm{eV}$ ), followed by the emission shoulder (blue, 95-99.5 eV) and the weak multiple scattering background (black, 71-84 eV). The onset of saturation can be connected with the stimulation cross-section. Emission above the band gap (red, 99.5-108 eV) is not observed at low intensities. The signal is connected with X-ray induced electronic excitations. 


\section{Methods}

\section{Experimental parameters.}

Photon Parameters. The experiments have been conducted during two separate runs at the freeelectron laser FLASH at Hamburg, Germany. The machine was operated at a central photon energy of about $115 \mathrm{eV}$. In one run, we recorded the spectral resolved data. The machine was operated at a repetition rate of $5 \mathrm{~Hz}$ with typically $30 \mathrm{fs}^{\text {pulses }}{ }^{17,27}$. We used beam line PG 2 with the 1200 lines/mm grating in zero order, thus reflecting the incident beam without dispersion ${ }^{28}$. To maximize the photon flux, a grazing angle of $3.5^{\circ}$ was used on the grating. The spot on the sample was round with about $45 \mu \mathrm{m}$ diameter, determined through measurements on a fluorescence screen and permanent imprints studied under a microscope. These parameters lead to fluences up to $1 \mathrm{~J} / \mathrm{cm}^{2}$. The incoming photon numbers have been measured for each shot by a gas monitor detector (GMD) ${ }^{29}$.

In another experimental campaign, the angular dependence of the stimulated emission signal was studied. Our setup was placed at beam line BL $2^{29}$. After an upgrade of the FLASH accelerator, a burst repetition rate of $10 \mathrm{~Hz}$ was now available, each including 100 pulses at $250 \mathrm{kHz}$. The pulse length was with about $50 \mathrm{fs}$ slightly longer and the spot size on the sample was adjusted to be about $40 \mu \mathrm{m}$ for compensation. With a gas attenuator, we set the fluence around $1 \mathrm{~J} / \mathrm{cm}^{2}$, the upper limit of the spectrally resolved data. 
Experimental Setup. Hydrogen passivated silicon (100) surfaces were scanned through the beam. The spectra have been recorded with a commercial Scienta XES 355 spectrometer ${ }^{30}$ using the 300 lines/mm grating. The counts have been detected by a multi-channel plate, phosphor screen combination that able to count single photons and is multi-hit capable. The detection window was centered at $92 \mathrm{eV}$ and the resolution set to $0.4 \mathrm{eV}$. The scattering plane was the horizontal plane of polarization of the incoming photons. The spectrometer was mounted at around $85^{\circ}$ to the beam $\left(15^{\circ}\right.$ to the sample surface), while the pulses impinged under an angle of $80^{\circ}$ to the surface.

For the angular dependence, we used an avalanche photodiode with an aluminum filter to block optical light. The incidence angle on the sample was $45^{\circ}$ while the detection angle was varied.

Analysis. With our spectrometer, we recorded around 40.000 single shots. The data was sorted, binned and the standard deviation of the averaged values were computed for each bin. With the photodiode, we analyzed 15.000 bursts.

During FEL irradiation, the emission from a plasma plume was optically visible at the sample and slight ablation was observed.

\section{Theoretical model.}

The instantaneous core hole density. As described in the main text, the instantaneous core hole density contains the main approximations in our theoretical treatment. Without stimulation, the 
core hole density is assumed to be constant inside a cylinder formed by the exciting radiation, outside it is assumed to be zero. The dimensions of this cylinder in the surface plane of the sample are given by the distribution of the incoming photons: the measured focal width and height $(w$ and $h$ ). The depth of the cylinder is taken as the tabulated absorption length of the incoming radiation $(\lambda)$. By choosing $1 / e$ dimensions for this volume, the integral number of core holes is the same as for the actual distributions and given through the number of incoming photons. We thus scale the core hole density to yield the same integrals and the same second moments as the actual distributions.

We treat the temporal distribution of the core holes as follows: Core hole decays during the pulse length reduce the instantaneous core hole density. The temporal distribution of the number of core holes in the sample at a given time $t, N_{C H}(t)$, is the solution of the following differential equation:

$$
\frac{d N_{C H}(t)}{d t}=N_{i n}(t)-\frac{N_{C H}(t)}{\tau_{l t}}
$$

with the temporal distribution of the exciting photons $N_{i n}(t)$ as the source term of this inhomogeneous differential equation and the core hole lifetime $\tau_{l t}$. For a temporal Gaussian pulse of incoming photons, the solution is the product of an exponential decaying function and an error function for the creation of core holes. To cast the analytical solution into the same approximation as above, where the core hole density (without stimulation) is constant during a specific time period, we analyze $N_{C H}(t)$ :

The integral of $N_{C H}(t)$ per incoming photon, i.e. the total number of core holes per photon 
present at each moment in time is proportional to $\tau_{l t}$. The longer the lifetime, the more core holes are present since they did not decay yet. The second central moment of $N_{C H}(t)$ for temporal Gaussian pulses is $\tau_{l t}^{2}+\tau_{p l}^{2}$ with the pulse length $\tau_{p l}$. The width of this function is approximated by the square root of the second moment. The constant function that yields the same integral over time $\left(\tau_{l t}\right)$, while it is zero outside a window as large as the square root of the second moment of $N_{C H}(t)$ is thus given by:

$$
T=\frac{\tau_{l t}}{\sqrt{\tau_{l t}^{2}+\tau_{p l}^{2}}}=\frac{1}{\sqrt{1+\frac{\tau_{p l}^{2}}{\tau_{l t}^{2}}}}
$$

This expression also accounts for the lowering of the number of core holes through spontaneous decay, as this is included in the core hole lifetime.

The instantaneous core hole density without stimulation is taken as constant, while stimulation annihilates core holes. The number of total stimulated photons is taken to be $K$-times bigger than observed (accounting for angular dependences and the finite detector acceptance and efficiency). The instantaneous core hole density $\rho_{c h}$ for the differential equation is thus given by:

$$
\begin{aligned}
\rho_{c h} & =p \cdot\left(N_{\text {in }}-K \cdot N_{\text {stim }, o b s}\right) \\
p & =\frac{T}{w h \lambda}
\end{aligned}
$$

The number of stimulating photons. The number of stimulating photons in the observation direction has been termed $N_{o b s}$. This value is given by the spontaneously emitted photons into the observation direction and the stimulated photons in the observation direction $N_{\text {stim,obs }}$. We introduce the acceptance and detection efficiency $A$ of our spectrometer, weighted with possible angular 
distributions of the spontaneous emission.

The total number of photons that are spontaneously emitted into the observed direction increases across the interaction region, starting from zero at the far end of the observed volume to the number of incoming photons $N_{i n}$ times the fluorescence yield $\omega_{f y}$ and the acceptance $\left(A \omega_{f y} N_{i n}\right)$. We approximate this increase with a constant across the interaction region. We take half the maximal value to yield the correct average value for a linear increase. This approximation ensures integrability and neglects the aspects around the temporal evolution, travel times of photons inside the interaction region etc.

Since stimulated emission annihilates core holes (and $K$-times more stimulated photons get emitted than are observed because of the finite acceptance of the detector), the number of spontaneously emitted photons gets reduced with higher stimulation. We thus introduce the source term for stimulated emission as:

$$
\begin{aligned}
N_{o b s} & =q \cdot\left(N_{\text {in }}-K \cdot N_{\text {stim }, o b s}\right)+N_{\text {stim }, o b s}, \\
q & =\frac{1}{2} A \cdot \omega_{f y}
\end{aligned}
$$

The full source term should in principal also reflect the spatial growth of the seeding spontaneous emission across the interaction region, which would lead to a slightly different non-linearity in the stimulated emission signal especially for low photon numbers. In reality, this effect is more than compensated by the bigger effect of the temporal mismatch of the seeding spontaneous emission (for near normal impinging irradiation, the seed appears at the same time across the whole 
interaction region in a time window of around $\sqrt{\tau_{l t}^{2}+\tau_{p l}^{2}} \approx 35 \mathrm{fs}$ (see above), while the travel time of the stimulated field across the $50 \mu \mathrm{m}$ long interaction region is already around $170 \mathrm{fs}$.) This can only be overcome by using a different geometry as proposed in the main text.

The full differential equation. In the differential equation for the growth of stimulated emission along the interaction region, we also treat the reabsorption of emitted photons explicitly, with a reabsorption length $L$. With the above introduced abbreviations, we thus solve:

$$
\begin{gathered}
\frac{d N_{\text {stim }, \text { obs }}}{d x}=\left(q \cdot N_{\text {in }}+(1-q K) \cdot N_{\text {stim }, \text { obs }}\right) \cdot\left(p \cdot N_{\text {in }}-p K \cdot N_{\text {stim }, o b s}\right) \cdot \sigma_{\text {stim }}-\frac{N_{\text {stim }, \text { obs }}}{L} \\
\text { with } \quad N_{\text {stim }, o b s}(x=0) \stackrel{!}{=} 0
\end{gathered}
$$

To get the total number of photons on the detector, we have to add the spontaneous emission, including the reduction through stimulated emission and the reabsorption along the interaction length $\mathrm{x}, \exp (-x / L)$ :

$$
N_{t o t, o b s}=2 q e^{(-x / L)} \cdot\left(N_{\text {in }}-K \cdot N_{\text {stim }, o b s}\right)+N_{\text {stim }, o b s}
$$

The solution and its limits. In the solution of the differential equation appear further combinations of parameters: $X:=x / L$ the ratio between the actual interaction length and the reabsorption length. This number is given by the experimental geometry and can thus be derived from tabulated values. $\Phi:=p \cdot L \cdot \sigma_{\text {stim }}$, a dimensionless number, that relates the excited volume $p^{-1}$ to a stimulation volume $L \cdot \sigma_{\text {stim }}$. The inverse value of $\Phi$ is connected to the number of photons to observe the non-linear increase in signal due to stimulated emission. 
The full solutions thus reads:

$$
\begin{aligned}
N_{\text {tot }, \text { obs }}= & 2 e^{-X} \cdot q N_{\text {in }}+\frac{1}{2 K \Phi(q K-1)} \cdot\left(1-2 e^{-X} q K\right) \\
\cdot & \left(1+\Phi N_{i n}(2 q K-1)-\sqrt{1+\Phi N_{i n}\left(\Phi N_{i n}+4 q K-2\right)}\right. \\
\cdot & \tanh \left(\frac{\sqrt{1+\Phi N_{i n}\left(\Phi N_{i n}+4 q K-2\right)} \cdot X}{2}\right. \\
+ & \left.\left.\operatorname{arctanh}\left(\frac{1+\Phi N_{i n}(2 q K-1)}{\sqrt{1+\Phi N_{i n}\left(\Phi N_{i n}+4 q K-2\right)}}\right)\right)\right)
\end{aligned}
$$

For small incoming photon numbers, the signal is proportional to the number of incoming photons, with purely spontaneous emission (with reabsorption) as linear limit:

$$
N_{\text {tot, obs }} \stackrel{N_{\text {in }} \rightarrow 0}{\longrightarrow} 2 e^{-X} \cdot q N_{\text {in }}=e^{-X} \cdot A \omega_{f y} N_{\text {in }}
$$

For very high numbers of incoming photons, the tanh in the formula nears unity and we obtain the following expression for the number of observed photons:

$$
N_{\text {obs }} \stackrel{N_{i n} \rightarrow \infty}{\longrightarrow} \frac{1}{K} \cdot N_{i n}
$$

This expression relates the saturation of stimulated emission to the geometry dependent $K$ factor that describes how much more stimulated emission happens in other directions than observed. As stimulated emission is a highly non-linear effect, this factor can vary largely, depending on the shape of the interaction region and how the observation direction is oriented relatively.

In our time-independent constant average discussion, the saturation of stimulated emission means a complete suppression of the Auger channel. In reality, Auger processes will still take place during the less intense beginning of the pulse, at the edges of the irradiated volume, as well 
as when the excited volume and the stimulated photons loose temporal overlap. Nevertheless, these contributions take out a constant fraction of the temporal and spatial evolution of stimulated emission and are thus linearly dependent on $N_{i n}$. Therefore, the convergence to a linear dependence is not altered.

\section{Fitting the model to the experimental data.}

We fit the obtained solution of the differential equation for $N_{t o t, \text { obs }}$ to the observed dependence of the signal on the incoming photon numbers recorded with the spectrometer as shown in Fig. 2.a and b. We determine the interaction length $x$ from the experimental geometry: with the incoming beam at $80^{\circ}$ to the surface, the observation direction at $15^{\circ}$ to the surface and an absorption length of the exciting radiation of $45 \mathrm{~nm}^{31}$, we estimate an interaction length of $171 \mathrm{~nm}$ and get $X=0.285$ with the reabsorption length $L=600 \mathrm{~nm}^{31}$. The parameters $q, K$ and $\Phi$ are then fitted to the data. We obtain $q=2.6 \cdot 10^{-11} \pm 0.3 \cdot 10^{-11}, K=1.100 \cdot 10^{10} \pm 0.003 \cdot 10^{10}$ and $\Phi=3.1 \cdot 10^{-11} \pm 0.3 \cdot 10^{-11}$. The fit error is given as one standard deviation.

Although we did not record many data points at very low intensities, the extrapolation of our data towards zero incoming photons can be readily done (Fig. 2.b). Besides the reabsorption factor $e^{-X}$ this value directly yields the parameter $q$ with a rather small error. The fitted value agrees well with our expectations and can be decomposed into the fluorescence yield ${ }^{8}\left(\omega_{f y}=3.8 \cdot 10^{-4}\right)$, the angular acceptance of our spectrometer ${ }^{30}$ of around $10^{-5}$ and the grating and detection efficiency 32 of $7 \cdot 10^{-3}$. 
The saturation limit $1 / K$ of the signal is close to the value of $q$. Due to the good data quality at higher photon numbers, we find a very small fit error. The value of $K$ signifies that much more stimulated photons are emitted into other directions than what we observe with our spectrometer. This finding is corroborated by our angular dependent study and can be further optimized by choosing different geometries, as described in the main text.

The fit parameter $\Phi$ is the main unknown in this study and accounts for the theoretical approximations as well as uncertainties in determining experimental parameters and further unconsidered non-linear effects. We decompose $\Phi$ into the factors $p=5.9 \cdot 10^{15} \mathrm{~m}^{-3}$ given through experimental parameters (focal width and height $45 \mu \mathrm{m}$, absorption length $45 \mathrm{~nm}{ }^{31}$, pulse length $30 \mathrm{fs}{ }^{27}$ and the core hole lifetime of $19 \mathrm{fs}^{33}$ ), the reabsorption length of $600 \mathrm{~nm}^{31}$ and the stimulation cross-section $\sigma_{\text {stim }}$. For the latter, we thus obtain a value of $8.8 \cdot 10^{-21} \mathrm{~m}^{2}$ which is about a factor of 30 bigger than the absorption cross-section. We have indications from rough calculations that the overlap between valence band and core states is actually about a factor 2-3 bigger than for conduction band and core states, which leads to a bigger stimulation than absorption cross-section. The remaining order of magnitude can be explained through the non-linearity of the effect. This will non-linearly enhance the signal from regions with higher excitation density, so that the effective focal size, excitation depth and duration are actually shorter than estimated. Therefore, we conclude that the fitted parameters agree reasonably well with our expectations.

31. Henke, B. L., Gullikson, E. M. \& Davis, J. C. X-ray interactions: Photoabsorption, scattering, transmission, and reflection at $\mathrm{E}=50-30,000 \mathrm{eV}, \mathrm{Z}=1-92$. Atom. Data Nucl. Data 54, 181 
(1993).

32. Kunnus, K. et al. A setup for resonant inelastic soft x-ray scattering on liquids at free electron laser light sources. Rev. Sci. Instrum. 83, 123109 (2012).

33. Hricovini, K. et al. Electronic-structure and its dependence on local order for H/Si(111)-(1x1) surfaces. Phys. Rev. Lett. 70, 1992-1995 (1993). 